Joachim Breckow und Albrecht M. Kellerer

\title{
Wirkungen kleiner Strahlendosen
}

\author{
Die neue Bewertung der Krebsmortalität der Überlebenden \\ von Hiroshima und Nagasaki führt zu einer erhöhten \\ Risikoschätzung für Strahlen-Kanzerogenese.
}

Als Wilhelm Conrad Röntgen am Neujahrstage des Jahres 1896 die ersten Sonderdrucke seiner Arbeit über eine neue Art von Strahlen verschickte, löste er eine Revolution aus, die innerhalb weniger Tage die Welt in Fieber versetzte. Unverzüglich wurden allerorts Röntgenröhren gebaut und betrieben, und es ist nur zu verständlich, daß die Möglichkeit schädlicher Wirkungen der neuen Strahlen nicht oder nur wenig bedacht wurde. Es dauerte nur wenige Monate, bis die ersten Hautschäden an den immer wieder zur Bildkontrolle benutzten Händen der Betreiber von Röntgenröhren deutlich wurden. Einige Jahre darauf wurden die ersten Fälle von Hautkrebs durch langandauernde wiederholte Röntgenbestrahlung bekannt und 1905 wurde erstmals über ein gehäuftes Auftreten von Leukämien bei Radiologen berichtet. Strahlenschutz beschränkte sich damals noch auf das Ziel, eine andauernde und mit erheblichen Dosen verbundene Exposition zu vermeiden. An mögliche Schäden durch kleine Dosen wurde nicht gedacht.

In den dreißiger Jahren bemalten hunderte von jungen Frauen, vor allem in den Vereinigten Staaten, im Akkord Ziffernblätter und Instrumentenskalen mit Radium-haltigen Leuchtfarben. Dabei spitzten sie die Pinsel mit dem Mund. Das dadurch inkorporierte Radium verursachte schon nach kurzer Zeit massive Kieferschäden und viele der Frauen gingen Jahre später an Knochentumoren zugrunde. Als ein junger Physiker auf die Strahlung des Radiums als mögliche Ursache hinwies, wurde er als Scharlatan zurechtgewiesen. Daß dieser Vorwurf von Marie Curie kam, wirft ein Schlaglicht auf die damals ganz lückenhafte Kenntnis über die biologischen Wirkungen ionisierender Strahlen. Für verläßliche Prinzipien des Strahlenschutzes fehlte noch jede Basis.

Für Jahrzehnte verblieb man im Zustand eines Enthusiasmus und Optimismus, der generell mit dem Glauben an positive Wirkungen ionisierender Strahlen einherging. Man schätzte hohe Radiumkonzentrationen nicht nur in Mineralwässern sondern pries sie sogar als hilfreich in Zahnpasten an. Sogenannte Radiumkissen wurden als Allheilmittel verkauft, und Röntgengeräte standen in allen besseren Schuhgeschäften.

Die Situation änderte sich, als wenige Jahre nach der Zerstörung von Hiroshima und $\mathrm{Na}$ gasaki unter den Überlebenden eine deutlich erhöhte Anzahl von Leukämieerkrankungen auftrat. Bereits 1927 war gezeigt worden, daß ionisierende Strahlen Mutationen hervorrufen können. Nun begann man zu verstehen, daß Leukämien das Resultat somatischer $\mathrm{Mu}$ tationen sein könnten, d.h. Veränderungen am genetischen Material einzelner Zellen. Obwohl das Problem strahleninduzierter Erbschäden, d.h. genetischer Veränderungen an Keimzellen, noch lange im Vordergrund blieb, begann die Problematik der Kanzerogenese durch ionisierende Strahlen mehr und mehr in den Blickpunkt öffentlichen Interesses und allgemeiner Besorgnisse zu rücken.

\section{Die Wirkung kleiner Strahlen- dosen}

Strahlenschäden nach hohen Dosen werden durch massiven Zelluntergang verursacht. Die Lethaldosis für den Menschen liegt bei einigen Gray (Gy), also etwa dem tausendfachen jener Dosis, die wir jährlich durch natürliche Exposition aufnehmen. Bei solch hohen Dosen werden, insbesondere in teilungsaktiven Geweben, soviele Zellen abgetötet, daß der Schaden nicht mehr durch Zellerneuerung kompensiert werden kann. Es kann zum akuten Strahlensyndrom kommen. Solche akuten oder nicht-stochastischen Strablenwirkungen, die auf massiven Zelluntergang zurückzuführen sind, lassen sich grundsätzlich vermeiden. Dazu muß die Exposition nur unterhalb bestimmter Dosisschwellen gehalten werden, bei denen Zellersatz bzw. -regenerierung in ausreichendem Maße stattfindet.

Zur Unterscheidung spricht man von stocbastischen Strablenwirkungen, wenn man sich auf
Veränderungen am genetischen Material einer Zelle, also gentoxische Wirkungen bezieht. Dabei unterscheidet man genetische und somatische Mutationen mit den möglichen Folgen von Erbschäden und Kanzerogenese.

\section{Erbschäden}

Ein schon seit Jahrzehnten diskutiertes Thema ist die Möglichkeit strahleninduzierter Erbschäden am Menschen. Daher war man sehr um den Nachweis einer Erhöhung der Raten für Erbschäden in Personengruppen mit erhöhter Strahlenexposition bemüht. Ein solcher Nachweis ist jedoch bislang noch in keiner Studie gelungen. Selbst in den 4 Jahrzehnten der epidemiologischen Beobachtung der Überlebenden in Hiroshima und Nagasaki und ihrer Nachkommen konnte keine statistisch signifikante Erhöhung der Raten für Erbschäden nachgewiesen werden. Die Schwierigkeit besteht darin, daß die Spontanrate hoch ist und stark von anderen Faktoren, wie zum Beispiel dem Alter der Eltern bei der Konzeption ihrer Kinder, abhängt.

Es ist jedoch sicher, daß ionisierende Strahlen auch Erbschäden verursachen. Man schließt aus Versuchen an Mäusen, daß eine einmalige Dosis von etwa 2 Gray zu einer Verdopplung von Erbschäden führt. Unter den Überlebenden von Hiroshima erhielten einige hundert Personen Expositionen dieser Größenordnung. Der Großteil der Überlebenden war jedoch geringeren Dosen von einigen zehntel Gray oder weniger ausgesetzt. In etwa $2 \mathrm{~km}$ Entfernung vom Epizentrum der Explosion war die durchschnittliche Strahlenexposition nurmehr wenige hundertstel Gray.

\section{Krebs}

Es ist die Besonderheit ionisierender Strahlen, daß durch einzelne geladene Teilchen beträchtliche Energie auf die Zelle übertragen werden kann. Die Strahlendosis ist lediglich ein statistischer Mittelwert der Energiedepo- 
sition im exponierten Gewebe, d.h. in einer Vielzahl von Zellen. Bei kleinen Dosen wird nur eine Minderheit von Zellen überhaupt von einem geladenen Teilchen durchquert. Die Anzahl der geschädigten Zellen ist proportional zu der Zahl der getroffenen Zellen und daher auch proportional zur Dosis. Eine Schädigung, die zur Tumorbildung führen kann, besteht in einer Veränderung an der zelleigenen DNA, die zwar nicht das Absterben der Zelle bewirkt, wohl aber eine Mutation und schließlich die Transformation und unkontrolliertes Teilungsverhalten der Zelle zur Folge hat. Der überwiegende Teil der DNA-Schäden wird durch zelluläre Reparatursysteme behoben, und nur ein sehr geringer Bruchteil transformierter Zellen kann die Immunschwellen durchbrechen und ungeregeltes Wachstum verursachen. Dies ändert aber nichts an der Tatsache einer einfachen Dosisproportionalität, sofern nicht auch Reparatursysteme und Immunreaktionen durch die Bestrahlung geändert sind. Bei kleinen Dosen ist dies jedoch unwahrscheinlich.

Angesichts solcher Wirkungsmechanismen ist davon auszugehen, daß es keinen Dosisschwellenwert gibt, unterhalb dessen ein Schaden ausbleibt und oberhalb dessen die Strahlung "gefährlich" ist.

Wenn man also annehmen muß, daß auch kleine Strahlendosen ein gewisses Risiko bergen, so ergibt sich die Notwendigkeit, die Höhe der Risiken abzuschätzen. Solche Abschätzungen beziehen sich auf die Erhöhung der Wahrscheinlichkeit an Krebs - als eines der mit Strahlung verbundenen Risiken - zu erkranken (Inzidenz) oder daran zu sterben (Mortalität).

Eine direkte Beobachtung der Erhöhung der Inzidenz- oder Mortalitätsraten aufgrund einer Strahlenexposition ist in Dosisbereichen von einigen $10 \mathrm{mGy}$, die gerade für den Strahlenschutz von Bedeutung sind, schwer oder gar nicht möglich, da sie weit unterhalb der spontan auftretenden Krebsraten bleiben. Außerdem sind die beobachteten Effekte nicht strahlenspezifisch, d.h. sie können durch verschiedene Faktoren ausgelöst werden und sind einer zuvor erfolgten Strahlenexposition nicht eindeutig ursächlich zuzuordnen.

Abschätzungen der Strahlenrisiken für diese Dosisbereiche beruhen also auf Extrapolationen von Ergebnissen epidemiologischer Studien, die sich auf höhere Dosen beziehen, und sie verlangen Hypothesen bezüglich der Art und Weise dieser Extrapolationen, die unbeweisbar bleiben. Überhöhte Risikoschätzungen können daher nicht widerlegt werden. Ebenso unmöglich ist es, extreme Gegenpositionen zu falsifizieren, die generell Schwellendosen für Strahlenkanzerogenese oder sogar allgemein positive Wirkungen kleiner Strahlendosen postulieren. Es ist daher nicht verwunderlich, daß sich in der Literatur Risikoschätzungen finden, die sich zum Teil um Größenordnungen voneinander unterscheiden [1].

Dennoch sind numerische Schätzungen sinnvoll und sogar notwendig, denn nur durch den Versuch quantitativer Bewertung gewinnt man Leitlinien, welche die Abschätzung und gegenseitige Minimierung unterschiedlicher Risiken und auch die stetige Verbesserung der Risikoabschätzung ermöglichen.

Die Revision der Atombombendosimetrie in Hiroshima und Nagasaki, die im folgenden erörtert wird, hat zu lebhaften Diskussionen und selbst zu scharfen Auseinandersetzungen über die bisherigen Risikoabschätzungen geführt. Die neuen Analysen sollten daher zum Anlaß genommen werden, die Risikoabschätzungen zu überprüfen.

\section{Das neue Dosimetriesystem}

Das Schicksal der Überlebenden der Atombombenangriffe auf Hiroshima und Nagasaki ist zum Gegenstand der wohl größten epidemiologischen Studie geworden, die jemals durchgeführt wurde. Die Ergebnisse dieser Studie sind in zahlreichen Berichten der ABCC (Atomic Bomb Casualty Commission) und später der RERF (Radiation Effects Research Foundation) niedergelegt; sie sind die wichtigste Quelle unseres Wissens über die Wirkungen kleiner Strahlendosen.

Durch die direkte Wirkung der Bomben wurden mehr als 200000 Menschen getötet. Vor der Zerstörung der beiden Städte war nicht angenommen worden, daß neben der Hitze und der Druckwelle auch die ionisierende Strahlung bedeutsam sein könnte. Immerhin gab es schon bald erste Dosismessungen und -berechnungen. Später wurden verschiedene Dosimetriesysteme entwickelt, von denen vor allem die sog. T-65 Dosimetrie (tentative dosimetry 1965) Anwendung fand. Die T-65 Dosimetrie ergab eine beträchtliche Neutronenkomponente der auf Hiroshima abgewor- fenen Uranbombe, deren Metallumhüllung die schnellen Spaltneutronen nur wenig abschirmte. Die Plutoniumbombe in Nagasaki setzte dagegen wenig Neutronen frei, da diese durch den zur Implosion benutzten konventionellen stark wasserstoffhaltigen Sprengstoff abgebremst und abgeschirmt wurden.

Die Annahme einer bedeutenden Neutronenkomponente der Strahlung in Hiroshima, nicht jedoch in Nagasaki, schien sich in der Form der Dosiswirkungsbeziehungen, beispielsweise für Leukämien, zu bestätigen. In Hiroshima ergab sich eine nahezu lineare Dosisabhängigkeit für die Leukämiewahrscheinlichkeit als Funktion der Dosis; in Nagasaki zeigte sich eine eher schwellenartige Abhängigkeit, d. h. eine erst bei höheren Dosen steiler werdende Funktion. Dies schien in Übereinstimmung mit der allgemeinen strahlenbiologischen Erfahrung, daß dicht ionisierende Strahlung, wie die durch Neutronen ausgelösten Rückstoßkerne, dosisproportional wirkt; locker ionisierende Strahlen, also vor allem Gammastrahlung, wird dagegen erst bei großen Dosen und dann überproportional wirksam.

Etwa 1975 wurden erste Zweifel an dieser Interpretation laut, da neue Transportrechnungen für Neutronen auf Fehler in der T-65 Dosimetrie hinwiesen. In langwieriger und aufwendiger Kooperation amerikanischer und japanischer Wissenschaftler wurde ein neues Dosimetriesystem entwickelt, das kürzlich veröffentlich wurde [2]. Die revidierte Dosimetrie, DS-86 (dosimetry system 1986), ergab als wichtigstes Resultat, daß die Neutronendosen in beiden Städten, vor allem aber in $\mathrm{Hi}_{\text {- }}$ roshima, weit geringer waren, als es den Ergebnissen der T-65 Dosimetrie entsprach. Dies lag zum Teil daran, daß für die T-65 Dosimetrie ein zu energiereiches Neutronenspektrum postuliert worden war; zum Teil aber auch an zusätzlichen Faktoren, wie etwa der früher vernachlässigten Luftfeuchtigkeit in Hiroshima.

Die Konsequenzen aus der Revision der Dosimetrie bezüglich des Neutronenanteils sind wenig bedeutsam für Nagasaki, da die Neutronenemission der dort abgeworfenen $\mathrm{Plu}$ toniumbombe schon früher als unerheblich angesehen wurde. Für Hiroshima dagegen ist die Verringerung der geschätzten Neutronendosen um etwa den Faktor 10 entscheidend, da früher ein Großteil der zusätzlichen Krebsfälle der Neutronenbestrahlung zugeschrieben wurde. 
Es lag daher die Aussage nahe, daß nach der Revision der Dosimetrie dieser Teil der Krebsfälle nunmehr zusätzlich der Gammastrahlung zuzuordnen sei; daher sollten sich die Risiken für Gammastrahlung stark erhöhen. Tatsächlich jedoch ergaben sich auch Veränderungen der Dosimetrie für Gammastrahlung. Gerade im Bereich kleiner Dosen, d.h. bei Entfernungen von mehr als einem Kilometer vom Epizentrum der Explosion, sind nach der revidierten Dosimetrie die Gammadosen deutlich höher als früher angenommen.

Das Fehlen der Neutronen in Hiroshima und die Kompensation durch die erhöhten Gammadosen hat zur Folge, daß für die Gesamtheit aller soliden Tumoren (also Krebs außer Leukämie) der Einfluß der Dosimetrierevision auf die Risikoschätzung bei kleinen Dosen nur gering ist. Insgesamt resultieren die Veränderungen der Dosimetrie aus einem komplexen Zusammenspiel verschiedener Faktoren. Die neuen Berechnungen ergeben etwa eine stärker abschirmende Wirkung von Häusern, andererseits jedoch eine höhere Transparenz des Körpers, d. h. eine geringere Abschirmung der tiefer liegenden Organe durch den Körper selbst.

Die erste und noch vorläufige RERF-Studie über den Einfluß der Dosimetrierevision auf die Risikoschätzungen für die Krebsmortalität [3] bezog alle soliden Tumoren auf Berechnungen der Äquivalentdosis im Gastrointestinaltrakt; damit ergaben sich um etwa $30 \%$ höhere Risikoabschätzungen als bei Anwendung der alten Dosimetrie; dabei geht man von einer relativen biologischen Wirksamkeit der Neutronen von 10 aus. Bei detaillierter Berechnung der Organdosen $[4,5]$ verschwindet bezüglich der Gammastrahlung der Einfluß der Dosimetrierevision fast vollständig. Für Leukämie dagegen ergibt sich hierdurch eine Erhöhung der Risikoabschätzung etwa um den Faktor 1,5.

Obwohl der Einfluß der Dosimetrierevision sich im Ganzen also als überraschend gering herausstellte, kam es durch andere Faktoren doch zu teilweise drastischen Erhöhungen der Risikoschätzungen gegenüber den früheren Angaben des „United Nations Scientific Committee on the Effects of Ionizing Radiation (UNSCEAR)“ [6] und der „International Commission on Radiological Protection $($ ICRP)" [7]. Die Gründe für diese Veränderungen der Risikoabschätzungen soll im folgenden dargestellt werden.

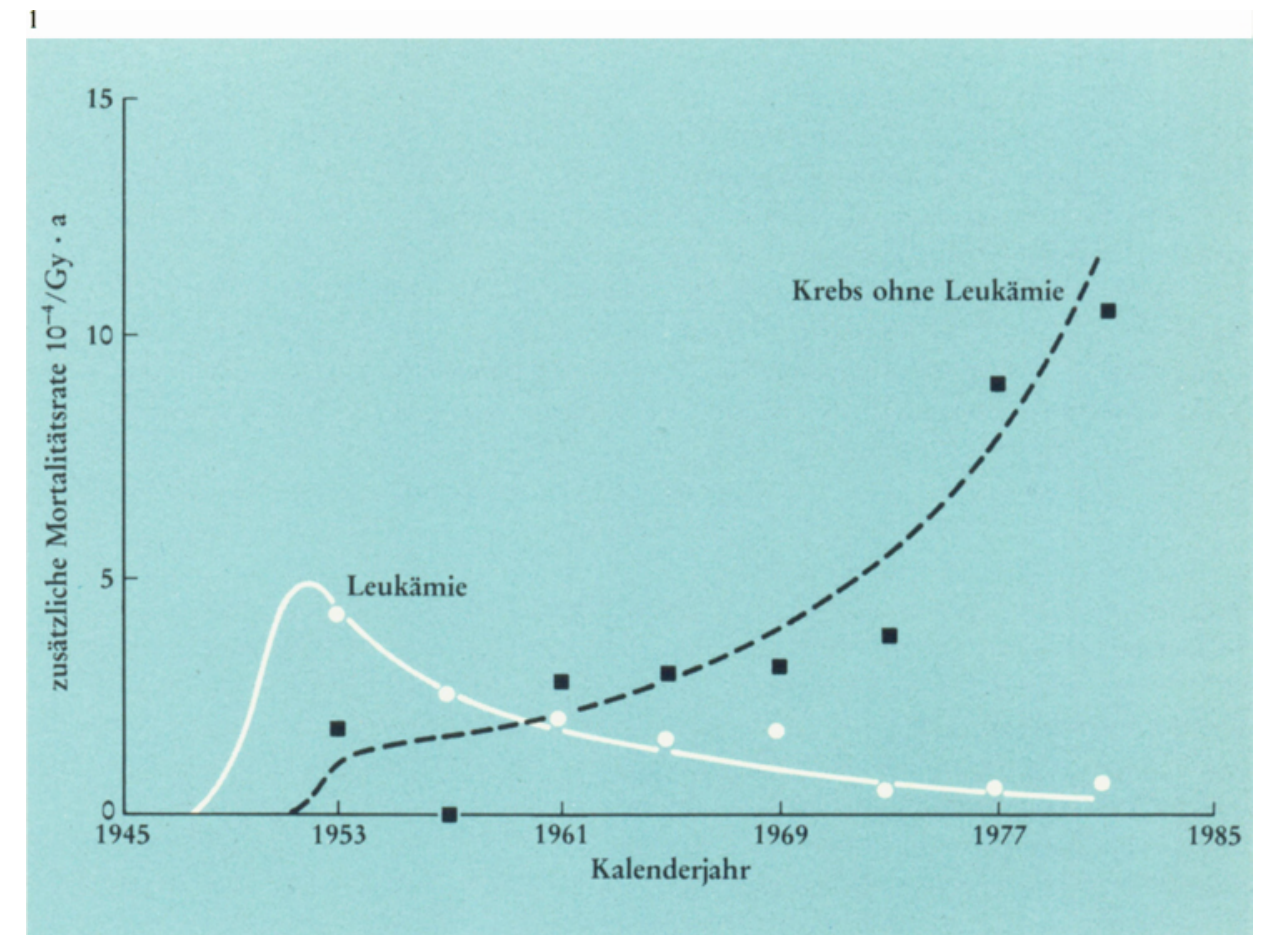

\section{Zeitabhängigkeiten von strahlen- induzierten Krebsmortalitätsraten}

Zeitliche Trends der Mortalitätsraten durch strahleninduzierte Tumore in Abhängigkeit von der Zeit nach einer Strahlenexposition wurden durch verschiedene epidemiologische Studien aufgezeigt (Abbildung 1). Während solide Tumore ein ähnliches Zeitverhalten aufweisen wie die spontanen altersspezifischen Tumorraten, treten strahleninduzierte Leukämien in einer charakteristischen zeitlichen Welle auf, die schon wenige Jahre nach Bestrahlung ein Maximum durchläuft und nach etwa 20 Jahren wieder verschwindet (Abbildungen 1 und 2).

\section{Relatives Risiko und absolutes Risiko}

Entsprechend den unterschiedlichen zeitlichen Verläufen beruht eine Vorhersage der über einen bestimmten Beobachtungszeitpunkt hinaus zu erwartenden zusätzlichen Krebsfälle auf sogenannten Lebenszeitprojektionsmodellen. Man unterscheidet zwischen dem Modell des relativen Risikos, das für die meisten soliden Tumore angenommen wird, und dem Modell des absoluten Risikos, das sich vor allem auf Leukämien bezieht. Gemäß dem Modell des relativen Risikos (Abbildung 2a) erhöhen sich die Tumorraten etwa 5 bis 10 Jahre nach Bestrahlung um einen bestimmten Faktor. Das jährliche $\mathrm{Zu}$ satzrisiko bleibt dann stets proportional zu
Abb. 1. Durch die Strahlenexposition hervorgerufene zusätzliche Krebsmortalität im Kollektiv der Atombombenüberlebenden. Jeder Punkt entspricht den Daten aus 4 Beobachtungsjahren; die interpolierenden Kurven dienen lediglich der leichteren Lesbarkeit; die Daten beinhalten eine sich zeitlich verändernde Altersverteilung des Kollektivs und sind daher nicht unmittelbar mit den Risikoabschätzungen zu verknüpfen (nach [8]).

den spontanen, altersspezifischen Tumorraten. Der Erhöhungsfaktor hängt also nicht von der Zeit nach der Exposition ab; er ist allerdings eine Funktion der Dosis, des Geschlechts und des Alters bei Bestrahlung. Es ist anzunehmen, daß der Faktor auch von der Art des Tumors abhängig ist; jedoch ist es schwierig, dies aufgrund der verfügbaren $\mathrm{Da}$ ten zu quantifizieren.

Die Daten zur Krebsmortalität der Atombombenüberlebenden, sowohl in Hiroshima als auch in Nagasaki, sind für solide Tumore über den gesamten Beobachtungszeitraum bis heute mit dem Modell des relativen Risikos vereinbar. Dieses Modell wird daher in fast allen numerischen Analysen benutzt.

Abbildung 2b stellt das Modell des absoluten Risikos dar, wie es aus den Leukämieinzidenzen in Hiroshima und Nagasaki erschlossen wurde. Ebenso wie für andere bösartige Er- 
krankungen erhöhen sich für Leukämien die spontanen Inzidenzen im Alter drastisch. Im Gegensatz zu den soliden Tumoren tritt jedoch schon bald nach einer Strahlenexposition eine Welle zusätzlicher Erkrankungen mit einem deutlichen Maximum auf. Nach ca. 20 bis 25 Jahren gleicht sich die Mortalitätsrate wieder der Rate für spontan auftretende Leukämien an.

\section{Die neuen Risikoschätzungen der Krebsmortalität}

Bevor die neuen Auswertungen im einzelnen erläutert werden, sei daran erinnert, daß die Beobachtung der Atombombenüberlebenden trotz des gewaltigen Umfanges dieser epidemiologischen Untersuchung Aussagen mit lediglich eingeschränkter statistischer $\mathrm{Ge}$ nauigkeit liefern kann. Die Gesamtzahlen für die Krebsmortalität verdeutlichen dies: Von den 75991 Überlebenden aus Hiroshima und Nagasaki, auf die sich die neuen RERFStudien beziehen, starben 5734 im Zeitraum von 1950 bis 1985 an soliden Tumoren, etwa 260 der Todesfälle, d. h. nahezu $5 \%$, werden aufgrund der statistischen Analyse der Strahlung zugerechnet. In der gleichen Gruppe und im gleichen Zeitraum starben 202 Menschen an Leukämie, etwa 80 dieser Leukämien, d.h. $40 \%$, wurden der Bestrahlung zugerechnet.

\section{Solide Tumore}

Für die neuen Analysen wurden von RERF erstmals Algorithmen für eine allgemeine Klasse von Risikomodellen benutzt. Für das Modell des relativen Risikos (also für die meisten soliden Tumore) wird angenommen, daß nach der anfänglichen Latenzzeit die proportionalen Erhöhungen der altersspezifischen Mortalitätsraten für Krebserkrankungen konstant bleiben und daß sie bis ins hohe Alter andauern. Aus dieser Extrapolation erhält man eine Abhängigkeit, die in Abbildung 3 als obere Kurve dargestellt ist und sich auf die linke Ordinate bezieht [3]. Die Berechnung ist für 6 Dosisgruppen durchgeführt. $\mathrm{Da}$ diese Daten alle Altersstufen und beide Geschlechter repräsentieren, sind in der angefügten Tabelle zusätzlich Skalierungsfaktoren angegeben. Es fällt auf, daß die Faktoren für Frauen diejenigen für Männer um mehr als das Doppelte überschreiten. Dies bedeutet jedoch nicht, daß Frauen in gleichen Zeiträumen nach Bestrahlung mehr zusätzliche Krebserkrankungen als Männer erleiden. Bei Frauen sind die altersspezifischen Inzidenzen

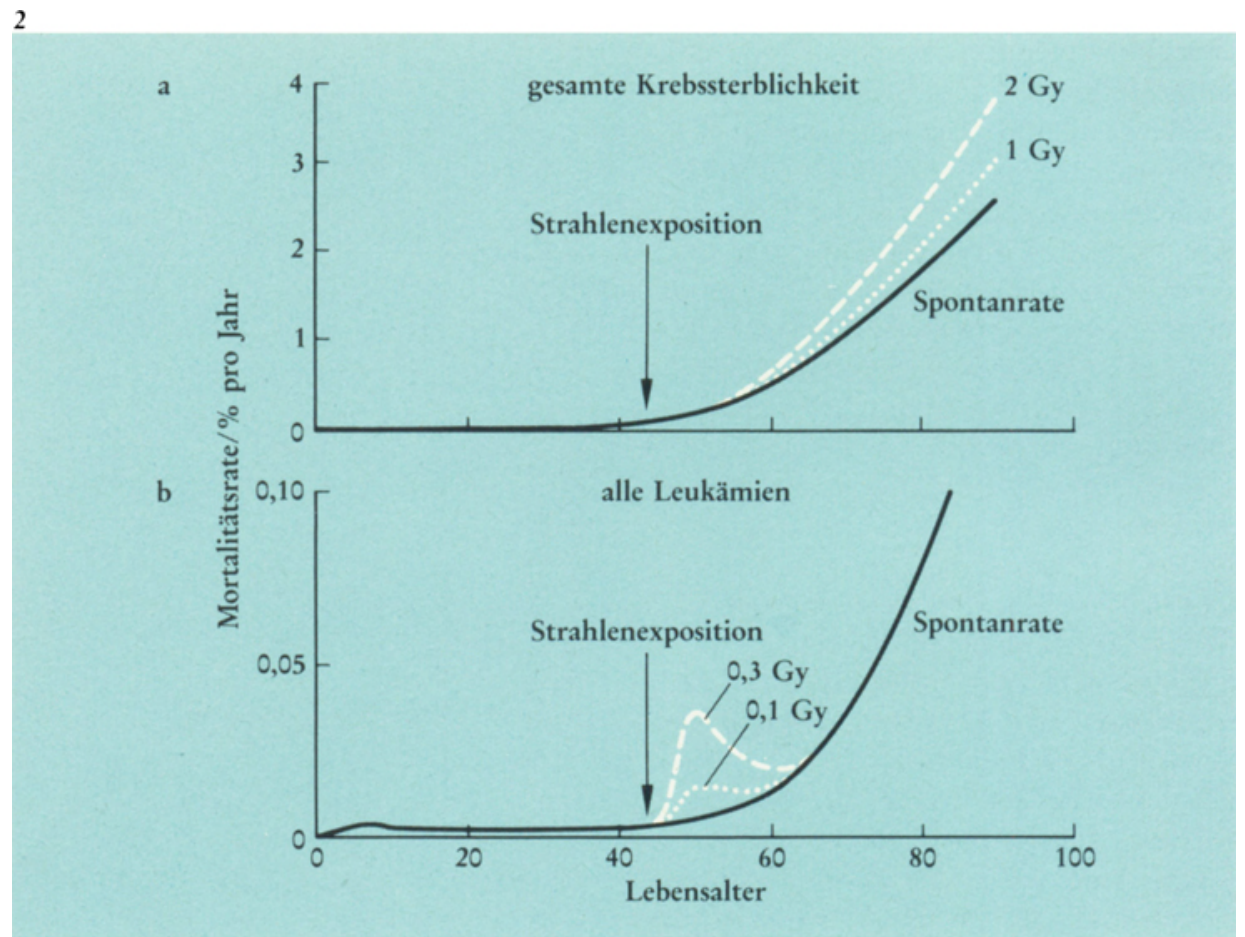

3

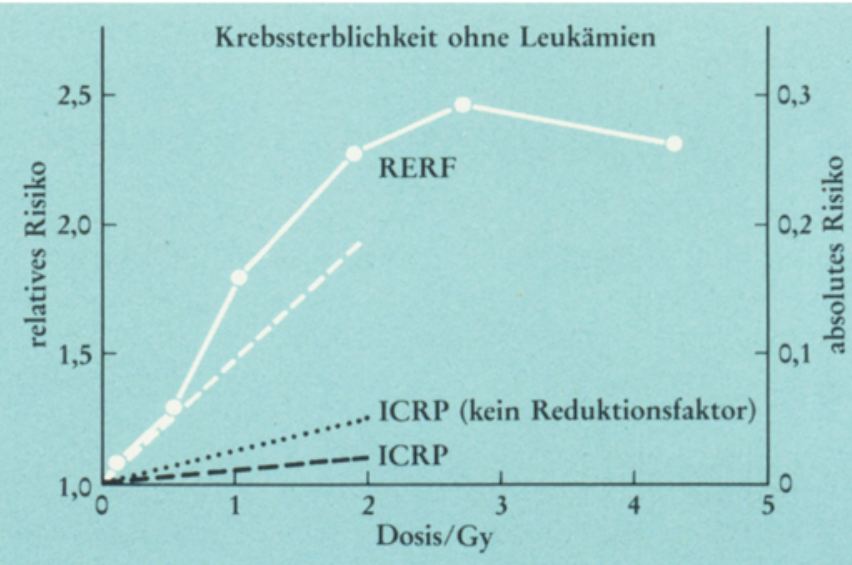

\begin{tabular}{|l|c|c|c|}
\hline \multirow{2}{*}{$\begin{array}{l}\text { Skalierungs- } \\
\text { faktoren }\end{array}$} & \multicolumn{3}{|c|}{ Alter bei Strahlenexposition } \\
\hline Frauen & 2,16 & 20 bis $35 \mathrm{a}$ & $>35 \mathrm{a}$ \\
\hline Männer & 0,97 & 1,29 & 0,71 \\
\hline
\end{tabular}

Abb. 2. Schematische Darstellung der Krebsmortalität nach dem Modell a) des relativen und b) des absoluten Risikos. Die durchgezogenen Kurven entsprechen der altersspezifischen Krebsmortalität der männlichen Bevölkerung der USA. Die Erhöhungen durch die angegebenen Strahlendosen entsprechen ungefähr den neuen Risikoschätzungen. Der Pfeil markiert den Zeitpunkt der Strahlenexposition.
Abb. 3. Dosisabhängigkeit des relativen und des absoluten Risikos der Krebssterblichkeit nach den neuen RERF-Analysen $[3,4,5]$. Zum Vergleich sind die Risikoschätzungen von ICRP [7] und die ohne Reduktionsfaktor resultierenden Werte angegeben. Die Ergebnisse von RERF beziehen sich auf das gesamte Kollektiv der Atombombenüberlebenden. Skalierungsfaktoren für verschiedene Altersgruppen sind in der Tabelle angegeben. 
spontaner Krebserkrankungen, zumindest im fortgeschrittenen Alter, etwa nur halb so hoch wie bei Männern. Näherungsweise führt eine Bestrahlung also zu der gleichen zusätzlichen Rate von Krebsfällen. Andererseits ergeben sich jedoch nach Summation über die gesamte Lebenszeit für Frauen doch mehr induzierte Krebstodesfälle. Wegen ihrer deutlich höheren Lebenserwartung sind Frauen dem Risiko, an Krebs zu erkranken, eben länger ausgesetzt.

Bei hohen geschätzten Dosen von mehr als 4 Gy, bei der nur wenige überlebten, haben die errechneten Werte für das relative zusätzliche Risiko wenig Relevanz für die Abschätzung von Strahlenrisiken. Bei den hohen geschätzten Dosen muß eine starke Verfälschung dadurch auftreten, daß mit größerer Wahrscheinlichkeit selektiv diejenigen überlebten, deren tatsächliche Dosen geringer als die Schätzungen waren. Eine entsprechende Unsicherheit könnte selbst noch im Bereich von 2 bis $3 \mathrm{~Gy}$ bedeutsam sein. Durch lineare Regression kann aus dem Anfangsteil der Kurve eine Erhöhung der Krebsmortalität um 60 bis $70 \%$ nach einer Dosis von 1 Gy Gammastrahlung erschlossen werden [3].

Die neueren Berichte von RERF [4, 5] gehen bei detaillierterer Berechnung der spezifischen Organdosen von einem relativen zusätzlichen Risiko für alle soliden Tumore von etwa $40 \%$ bei $1 \mathrm{~Gy}$ aus. Das entsprechende absolute zusätzliche Risiko über die gesamte Lebenszeit, der sog. Risikokoeffizient, liegt bei etwa 0,12/Gy (Abbildung 3, rechte Ordinate). Dies bedeutet 120 zusätzliche Fälle pro 1000 mit $1 \mathrm{~Gy}$ exponierte Personen [5].

Eine lebhafte Diskussion entzündete sich an den neuen Risikoschätzungen schon bald nach den ersten neuen Berichten von RERF, weil eine beträchtliche Erhöhung gegenüber den früheren Schätzungen von UNSCEAR [6] und ICRP [7] deutlich wurde (Abbildung 3). Die untere Gerade gibt die früher postulierte Abhängigkeit wieder, der ein geschätztes absolutes Risiko von 0,01 pro Gray entspricht. Man erkennt einen überraschend großen Unterschied zu den neuen Daten von RERF um etwa den Faktor 10.

Ein Faktor von nahezu 2 entspricht den zusätzlichen der Bestrahlung zuzurechnenden Krebstodesfällen, die seit 1975 (dem Enddatum für bisherige Risikoschätzungen) bis zum Jahre 1985 aufgetreten sind. Der beträchtliche Zuwachs erklärt sich dadurch,

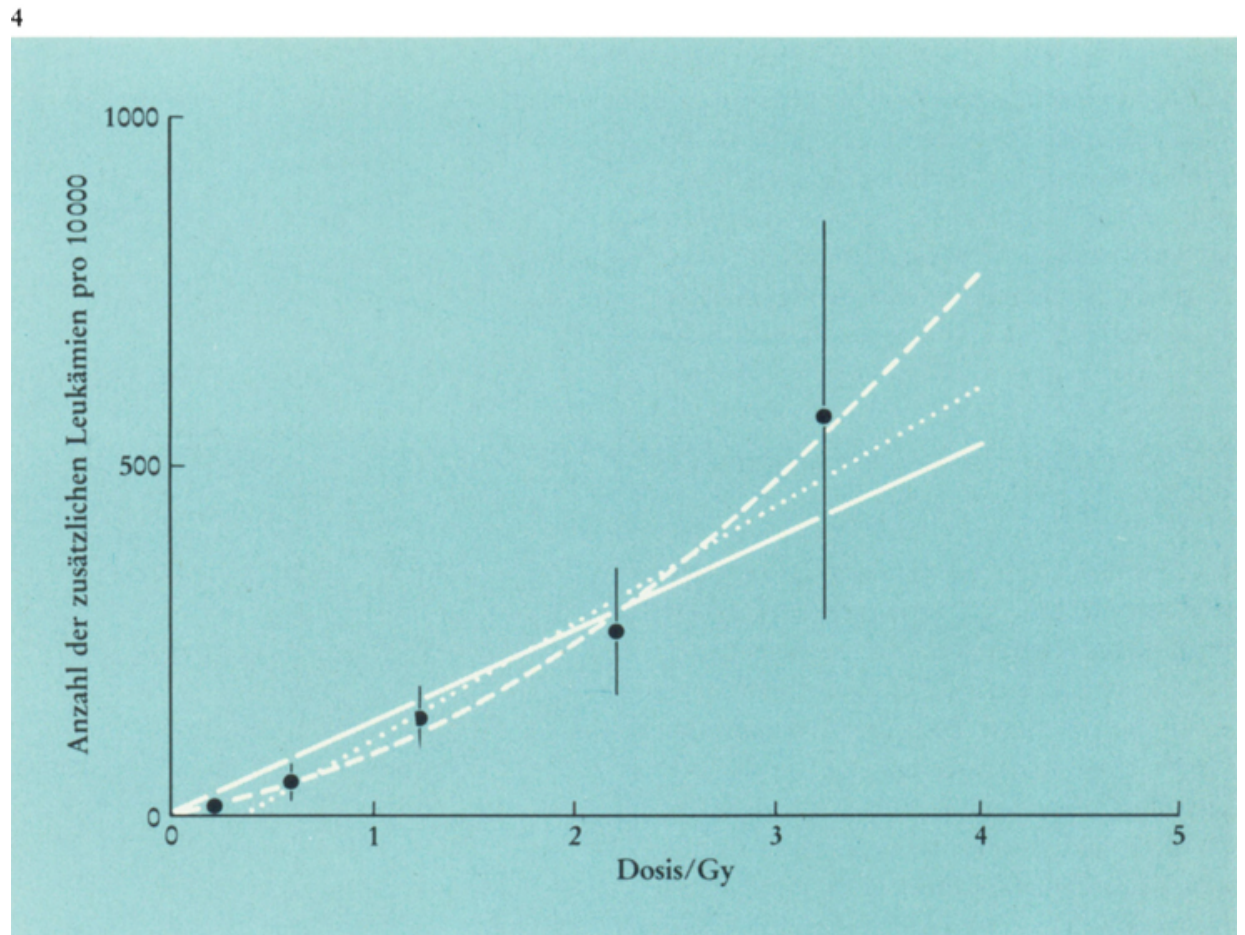

daß ein großer Teil der Überlebenden nunmehr in ein höheres Alter gekommen ist, in dem auch die spontanen Krebsraten steil ansteigen. Dies fällt besonders ins Gewicht, weil die in jüngerem Alter Exponierten höhere Empfindlichkeit, d.h. insgesamt größere proportionale Erhöhungen der Krebsmortalität, aufweisen (siehe Tabelle zu Abbildung 3). Vor 1975 traten für diese Altersgruppen nur wenige Krebserkrankungen auf, wenn man von Leukämien absieht.

Die als Kinder Bestrahlten haben noch nicht das Lebensalter erreicht, in dem die Krebsmortalität statistisch faßbar wird. Sollte, allen Erwartungen entsprechend, auch ihre Empfindlichkeit sich als hoch erweisen und sollten die Erhöhungen über die gesamte Lebenszeit fortdauern, so wäre mit einem weiteren Ansteigen der insgesamt zu erwartenden und der Bestrahlung zuzuschreibenden Krebstodesfälle um noch einmal etwa den Faktor 2 zu rechnen. In die RERF-Analysen, die eine solche andauernde Erhöhung der Mortalitätsraten postulierten, ist auch dieser Faktor bereits eingeschlossen.

Ein bedeutsamer Unterschied zwischen den früheren Analysen und den neueren Studien basiert auf den folgenden früheren Überlegungen von UNSCEAR und ICRP: demnach sollte ein Reduktionsfaktor von etwa 2,5 der Tatsache Rechnung tragen, daß die beobachteten Daten sowohl für solide Tumore als auch für Leukämien sich auf relativ hohe Do-
Abb. 4. Mögliche Abhängigkeiten der Leukämiesterblichkeit von der Dosis. Die Daten sind der Abbildung 5 für den Dosisbereich zwischen 0,1 Gy und 3,5 Gy entnommen. Eine konservative Risikoabschätzung für kleine Dosen basiert auf der linearen Extrapolation (durchgezogene Kurve). Strahlenbiologische Untersuchungen geben dagegen Hinweise auf eine linearquadratische Dosiswirkungsbeziehung (gestrichelte Kurve). Es gibt wenig Anhaltspunkte für die Annahme einer Dosisabhängigkeit mit Dosisschwelle (gepunktete Kurve).

sen beziehen. Nach allgemeinen strahlenbiologischen Erfahrungen ergeben sich für Gammastrahlen linear-quadratische Abhängigkeiten. Solche Abhängigkeiten entsprechen verringerter Wirkung pro Dosiseinheit bei kleinen Dosen (Abbildung 4).

Heute ist ein Reduktionsfaktor bei der Extrapolation von hohen Dosen zu den im Strahlenschutz relevanten geringen Dosen weniger leicht begründbar. Die Dosisabhängigkeiten für Krebsmortalität in Hiroshima und Nagasaki sind nach den Analysen von RERF nicht mehr signifikant verschieden. Im analysierbaren Dosisbereich erscheinen sie, wie aus Abbildung 3 ersichtlich, linear. Ein - möglicherweise beträchtlicher - Reduktionsfaktor könnte vorliegen, ist aber nicht mehr aufzeigbar. Obwohl selbst die extreme Annahme un- 
widerlegbar bleibt, daß bei kleinsten Dosen unterhalb einer Schwelle keinerlei Erhöhung der Krebsinzidenz hervorgerufen werde (Abbildung 4), kann eine vorsichtige Extrapolation sich dennoch einfacher Linearität bedienen und auf die Annahme eines Reduktionsfaktors verzichten. Ohne den Reduktionsfaktor entsprächen die früheren ICRP-Schätzungen den gepunkteten Geraden in Abbildung 3 und 5 .

\section{Leukämien}

Im letzten Jahrzehnt sind wenig zusätzliche der Bestrahlung zugerechnete LeukämieFälle aufgetreten (Abbildung 1). Die Analyse bezieht sich daher fast ausschließlich auf die schon beobachteten Fälle, ohne das Problem der Projektion in die Zukunft. Die in den verschiedenen Studien von RERF errechneten Dosisabhängigkeiten stimmen gut überein; sie sind, auf das Gesamtrisiko umgerechnet, in Abbildung 5 dargestellt und ergeben einen Risikokoeffizienten von etwa 0,01/Gy. Auch diese Abhängigkeiten lassen sich mit den Schätzungen von ICRP vergleichen.

\section{Schlußfolgerungen}

Die neue Bewertung der Krebsmortalität der Überlebenden von Hiroshima und Nagasaki führt aus mehreren Gründen zu erhöhter Risikoschätzung für Strahlenkanzerogenese. Die Revision der Dosimetrie hat eine bedeutsame Änderung insofern erbracht, als nun kein direkter Hinweis für schwellenartige Dosisabhängigkeiten der Krebsmortalität bei locker ionisierenden Strahlen mehr besteht. Ein Reduktionsfaktor für die Extrapolation aus dem Bereich hoher Dosen zu kleinen Dosen ist lediglich durch allgemeine strahlenbiologische Argumente jedoch nicht mehr durch die Mortalitätsdaten selbst begründbar. Eine konservative Risikoschätzung wird sich daher keines Reduktionsfaktors bedienen. Dies allein führt zu einer Erhöhung der Risikoschätzung etwa um den Faktor 2,5.

Von großer Bedeutung sind die großen relativen Erhöhungen der Tumorraten für die in jugendlichem oder kindlichem Alter bestrahlten Überlebenden. Sollten diese Erhöhungen über das ganze Leben fortdauern - und dies wird erst nach Fortführung der epidemiologischen Studie bis weit über das Jahr 2000 hinaus deutlich werden - ergeben sich hohe absolute Risikofaktoren für Strahlenexposition in jugendlichem Alter. Dies wird das zentrale Problem der fortdauernden Beobachtungen

5

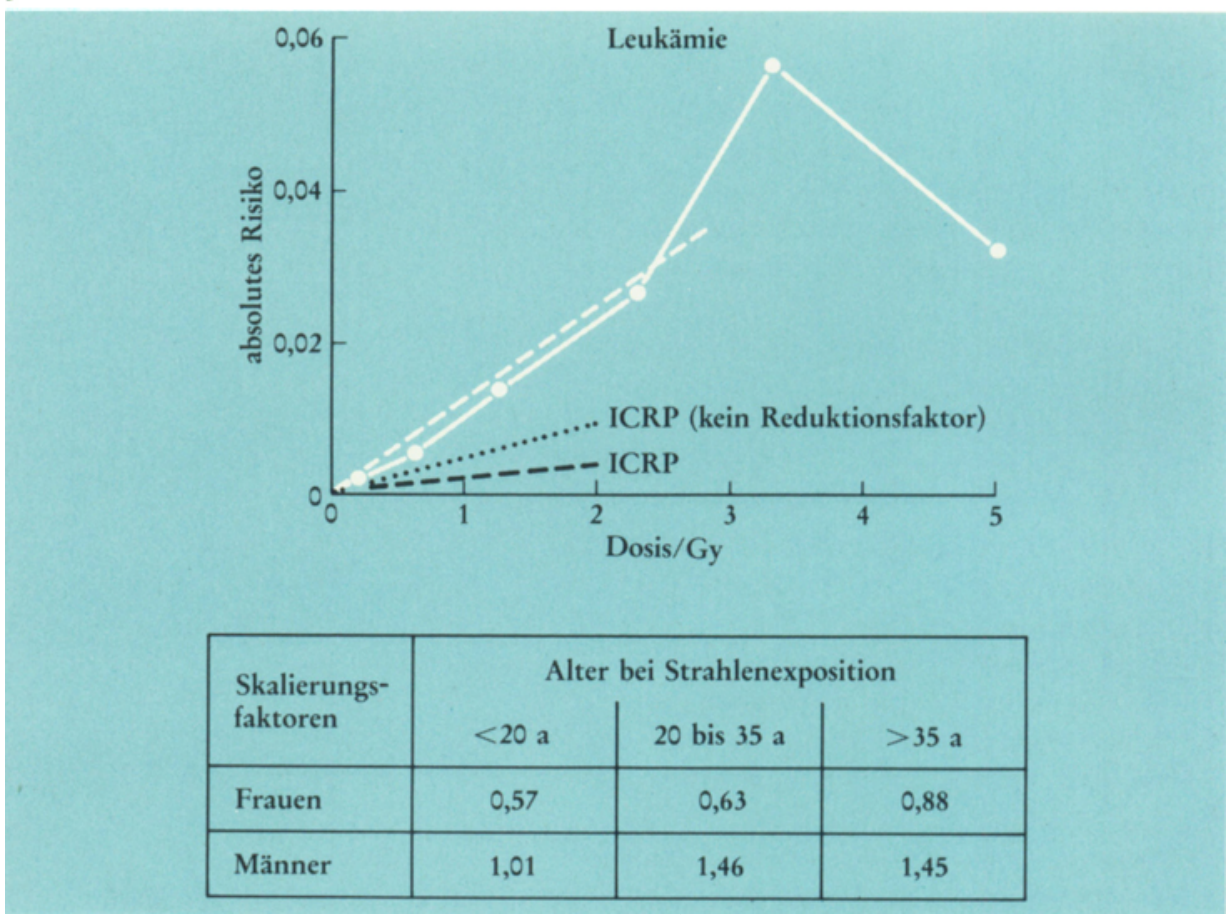

sein. Die Neubewertung der Risikoabschätzungen für berufliche Strahlenexposition bleibt jedoch von dieser Frage weitgehend unberührt.

Die angeführten Zahlenwerte sind mit großen Unsicherheiten behaftet, da sie trotz des Umfangs der Studie auf relativ wenigen zusätzlichen Todesfällen beruhen. Noch gröBere Unsicherheit besteht im Hinblick auf strahleninduzierte Erbschäden, für die sich eine statistisch signifikante Erhöhung bisher in den Nachkommen der Überlebenden von Hiroshima und Nagasaki nicht nachweisen ließ. Man ist hier nach wie vor auf Daten aus Tierversuchen angewiesen, die auf einen Risikokoeffizienten in der Größenordnung von 0,01/Gy hindeuten. Eine Erörterung dieser Problematik liegt ebenso außerhalb des Rahmens dieses Berichts wie die Diskussion der wichtigen neuen Erkenntnisse über die besondere Empfindlichkeit des sich entwikkelnden Zentralnervensystems in der Embryonalphase zwischen etwa der 8. und 15 . Woche der Schwangerschaft.

Es bleibt die Frage, ob es nicht gegen wissenschaftliche Prinzipien verstößt, Risikoschätzungen zu erörtern, die auf hypothetischer Extrapolation aus dem Bereich hoher Dosen zu viel kleineren Dosen beruhen. Die Antwort ist, daß bei aller bestehender Unsicherheit die Risikoabschätzungen als plausible Werte anzusehen sind, die zwar Überschätzungen sein können aber nicht sein müssen.
Abb. 5. Dosisabhängigkeit des absoluten Risikos der Leukämiesterblichkeit nach den neuen RERF-Analysen [3, 4, 5]. Zum Vergleich sind die Risikoschätzungen von ICRP [7] und die ohne Reduktionsfaktor resultierenden Werte angegeben. Die Ergebnisse von RERF beziehen sich auf das gesamte Kollektiv der Atombombenüberlebenden; sie sind aus den publizierten $\mathrm{Da}$ ten unter der Annahme einer mittleren Zeit „at risk“ von 32 Jahren abgeleitet. Skalierungsfaktoren für verschiedene Altersgruppen sind in der Tabelle angegeben. Die ICRP-Schätzung bezieht sich auf die Altersverteilung einer erwachsenen Bevölkerung. 
Die Prinzipien des Strahlenschutzes müssen auch dann verantwortbar bleiben, wenn die erhöhten Risikoabschätzungen keine Überschätzungen sind. Das System der Dosisgrenzwerte, kombiniert mit dem Grundprinzip der vernünftigen Minimierung oder Vermeidung unnötiger Strahlenexpositionen, sollte auch heute die Gewähr bieten, daß die Risiken gering sind gegenüber anderen, weit höheren und zum großen Teil vermeidbaren Krebsrisiken.

Es scheint jedoch jetzt noch weniger als in der Vergangenheit verantwortbar, beruflich strahlenexponierte Personen regelmäßig bis zu den jährlichen Dosisgrenzwerten zu exponieren. Um solche weit über dem Durchschnitt liegende Expositionen selbst für kleinere berufliche Gruppen zu vermeiden, verlangt die kürzlich novellierte Strahlenschutzverordnung nun, über das generelle Minimierungsangebot und das jährliche Limit von $50 \mathrm{mSv}$ hinaus, für beruflich strahlenexponierte Personen die Lebenszeitdosis auf $400 \mathrm{mSv}$ zu begrenzen.

In Großbritannien und Schweden wurden die entsprechenden Regelungen getroffen, daß die Exposition durch berufliche Tätigkeit im Mittel eine Jahresdosis von $15 \mathrm{mSv}$ nicht überschreiten dürfe. In naher Zukunft wird eine gemeinsame europäische Konvention nötig werden. Eine solche Regelung sollte sich auf die spätestens $1990 \mathrm{zu}$ erwartenden neuen Empfehlungen der ICRP stützen.

Es wird gelegentlich festgestellt, numerische Risikoabschätzungen seien angesichts bestehender Unsicherheiten nicht gerechtfertigt; sie führten lediglich zur maßstabslosen Angst vor noch so kleinen Dosen ionisierender Strahlen. Dem ist zu entgegnen, daß einseitiger Überbewertung gewisser Risiken nur durch vergleichende Abschätzungen begegnet werden kann. Notwendig ist daher nicht das Aufgeben der im Strahlenschutz entwikkelten quantitativen Konzepte, sondern ihre Übertragung auch auf andere Gebiete. Bezüglich chemischer Kanzerogene ergibt sich neuerdings ein Konsensus der Toxikologen, daß für die Wirkung gentoxischer Substanzen, d.h. für stochastische Wirkungen wie Kanzerogenese und Erbschäden, keine Schwellenwerte der Dosis oder Konzentration angenommen werden können. Bewertung und die Akzeptanz von Risiken müssen sich also auf numerische Abschätzungen und schließlich auf politische oder gesellschaftliche Entscheidungen stützen. Was bisher ein besonderer und scheinbar überkonservativer Ansatz des Strahlenschutzes zu sein schien, wird nunmehr auf ein viel weiteres Gebiet bezogen.

\section{Literatur}

[1] H. G. Paretzke, Phys. Bl. 45, 16 (1989).

[2] US-Japan Joint Reassessment of Atomic Bomb Radiation Dosimetry in Hiroshima and Nagasaki, Final Report, Vol. 1 (W. E. Roesch, Ed.), Radiation Effects Research Foundation, Hiroshima, 1987.

[3] D. L. Preston, D. A. Pierce, Radiat. Res. 114, 437 (1988).

[4] Y. Shimizu, H. Kato, W. J. Schull, D. L. Preston, S. Fujita, D. A. Pierce, Radiat. Res. 118, 502 (1989).

[5] Y. Shimizu, H. Kato, W. J. Schull, Life Span Study Report 11. Part 2. Cancer Mortality in the Years 1950-1985 based on the recently revised doses (DS86). Technical Report, RERF TR 5-88, Radiation Effects Research Foundation, Hiroshima (1988).

[6] Sources and Effects in Ionizing Radiation. United Nations Scientific Committee on the Effects of Ionizing Radiation (UNSCEAR), United Nations Sales Publication Number E.77.IX.1 New York, 1977.

[7] Annals of the ICRP, Publication 26. Recommendations of the International Commission on Radiological Protection. Pergamon Press, Oxford 1977.

[8] W. K. Sinclair, Radiat. Res. 112, 191 (1987).

Prof. Dr. A. M. Kellerer und Dr. J. Breckow, Institut für Medizinische Strahlenkunde der Universität Würzburg, Versbacher Straße 5, D-8700 Würzburg. 